\title{
Editorial
}

\section{Photobiomodulation 2014}

\author{
Timon Cheng-Yi Liu, ${ }^{1}$ Quan-Guang Zhang, ${ }^{2}$ and Lutz Wilden ${ }^{3}$ \\ ${ }^{1}$ Laboratory of Laser Sports Medicine, South China Normal University, Guangzhou 510006, China \\ ${ }^{2}$ Institute of Molecular Medicine and Genetics, Medical College of Georgia, Georgia Regents University, Augusta, GA 30912, USA \\ ${ }^{3}$ Privatpraxis für Hochdosierte Low Level Lasertherapie, Wilden, L. Kuralle 16, 94072 Bad Füssing, Germany \\ Correspondence should be addressed to Timon Cheng-Yi Liu; liutcy@scnu.edu.cn
}

Received 30 September 2014; Accepted 30 September 2014; Published 9 November 2014

Copyright (C) 2014 Timon Cheng-Yi Liu et al. This is an open access article distributed under the Creative Commons Attribution License, which permits unrestricted use, distribution, and reproduction in any medium, provided the original work is properly cited.

It is our great pleasure to present this special issue of this journal. The stories of photobiomodulation (PBM) of a low level laser irradiation or monochromatic light (LLL) continued in this issue since its first annual issue published in 2012. We have selected eleven papers: two review papers making progress in understanding PBM mechanism, four original papers about cellular PBM, one original paper about clinical PBM, three original papers about photodynamic therapy, and one original paper about visual imagine operation of athletes. These eleven articles offered advancements in both understanding and optimizing potential new technologies.

Among these eleven articles, the cross-talking between the negative feedback and functions is very interesting. As T. C.-Y. Liu et al. have pointed out, there is a functionspecific homeostasis (FSH), a negative feedback mechanism for a function to be perfectly performed. A function can be finely classified into a normal function in its FSH and a dysfunctional function far from its FSH. A PBM can be then finely classified into a direct PBM (dPBM) to modulate a dysfunctional function and an indirect PBM (iPBM) to upgrade a normal function. With its negative feedback mechanism, a normal function can resist external disturbance under its threshold so that it resists a dPBM. Far from its negative feedback mechanism, a dysfunctional function is sensitive to its external disturbance so that a LLL may selfadaptively modulate it until it becomes a normal function in a $\mathrm{dPBM}$ way. A cell responds to an external signal with its intracellular signal transduction pathways. A cellular normal function not only can resist its external disturbance, but also can resist the activation of other signal transduction pathways to maintain the full activation of its specific signal transduction pathways (normal function-specific signal transduction pathways, NSPs) so that it does not respond to some external signals under their respective thresholds. However, almost all the signal transduction pathways are partially activated for a cellular dysfunctional function so that the cell can respond to any external signals. A normal function may have $n$ NSPs which are called redundant pathways with one another, and the normal function maintained by the synergistic activation of $n$ NSPs is called the $n$ th-order normal function. In other words, the NSPs maintaining a normal function are very sparse, but the signal transduction pathways maintaining a dysfunctional function are extraordinarily dense. The activation of each signal transduction pathway needs adenosine $-5^{\prime}$-triphosphate (ATP). This is why the ATP level of a cell with its dysfunctional function is lower than the one of the same cell with a normal function. A cellular dPBM may promote the microenvironment-allowed activation of a partially activated NSP of a dysfunctional function until it is fully activated and then the normalization of the dysfunctional function so that it may also increase the ATP level. A cellular iPBM may promote the microenvironmentallowed activation of partially activated redundant NSPs of a normal function until they are fully activated so that the normal function is upgraded. Whether a cellular function is a normal or dysfunctional function and the activation of which partially activated NSPs is promoted by a PBM depend on the cellular microenvironment which then depends on the global action of an organism so that a microenvironment dependent cellular PBM is in agreement with an actiondependent global PBM.

C.-P. Zhang et al. have studied effects of a low intensity $\mathrm{He}-\mathrm{Ne}$ laser irradiation on the proliferative potential and cell-cycle progression of myoblasts. Primary myoblasts were 
derived from hindlimb muscles of neonatal Wistar rats and cultured in Ham's F-10 nutrient mixture supplemented with $0 \%, 10 \%$, and $20 \%$ fetal bovine serum (FBS), respectively. They found the promotion of the PBM on the proliferation, cyclin A, and cyclin D of the serum-free myoblasts. As they have observed, there was dysfunctional proliferation of myoblasts in Ham's F-10 nutrient mixture with 0\% FBS. Therefore, the observed PBM just played a dPBM role.

S. Li et al. have studied PBM for cobalt chloride- $\left(\mathrm{CoCl}_{2}-\right)$ induced hypoxic dysfunction of rhesus monkey choroidretinal (RF/6A) cells by $670 \mathrm{~nm}$ light-emitting diode (LED) irradiation. They observed the proliferation of $\mathrm{RF} / 6 \mathrm{~A}$ cells in Dulbecco's minimal essential medium (DMEM)/F12 with $10 \%$ FBS resisted the $\mathrm{CoCl}_{2}$ at $100 \mu \mathrm{mol} / \mathrm{L}$ so that it was in its normoxia proliferation-specific homeostasis (nPISH), and they found no dPBM on its cytochrome $\mathrm{C}$ oxidase (COX) activity and ATP concentration. They further observed $\mathrm{CoCl}_{2}$ at $200 \mu \mathrm{mol} / \mathrm{L}$ disrupted the $\mathrm{nPlSH}$ and induced the dysfunctional proliferation, and they found a dPBM may completely recover the normal proliferation, but the COX activity and ATP concentration were only partially recovered. In other words, the established proliferation-specific homeostasis ( $\mathrm{PlSH}$ ) was different from the $\mathrm{nPISH}$ and may be called hypoxic $\mathrm{PlSH}$ (hPlSH). The $\mathrm{nPlSH}$ and hPlSH maintain the same normal proliferation, but their mechanisms are different from each other.

$\mathrm{X}$. Chen et al. have studied the effects of $808 \mathrm{~nm}$ LED light pretreatment of hypoxic primary mouse cortical neurons. They observed the LED light did not affect the COX activity and ATP concentration of the neurons in DMEM/F12 with $10 \%$ FBS and $10 \%$ horse serum so that the proliferation may be the normal proliferation in its $\mathrm{nPISH}$. The proliferation in its $\mathrm{nPlSH}$ could not resist $\mathrm{CoCl}_{2}$ at $200 \mu \mathrm{mol} / \mathrm{L}$ but the LED light pretreated neurons could. It suggested LED light pretreatment enhanced the $\mathrm{nPISH}$ so that the enhanced nPISH $(\mathrm{ePlSH})$ could resist $\mathrm{CoCl}_{2}$ at $200 \mu \mathrm{mol} / \mathrm{L}$. The LED light pretreatment just played an iPBM role. The iPBM did not affect the COX activity and ATP concentration. The normal proliferation in its ePISH could resist $\mathrm{CoCl}_{2}$ at $200 \mu \mathrm{mol} / \mathrm{L}$ but its COX activity and ATP concentration could not.

\section{Acknowledgment}

We are thankful to the contributing authors who have provided compelling work that will serve a springboard for thoughtful discussions and fuel for future studies.

Timon Cheng-Yi Liu

Quan-Guang Zhang

Lutz Wilden 

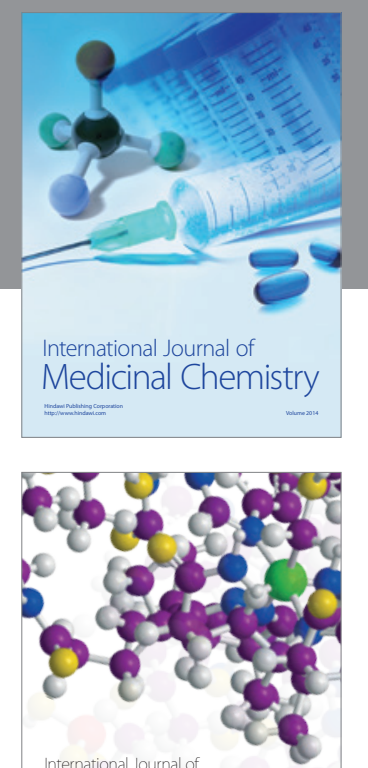

\section{Carbohydrate} Chemistry

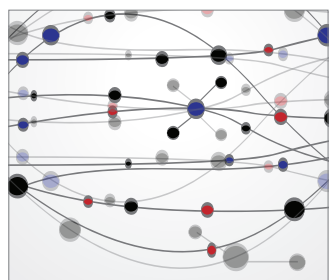

The Scientific World Journal
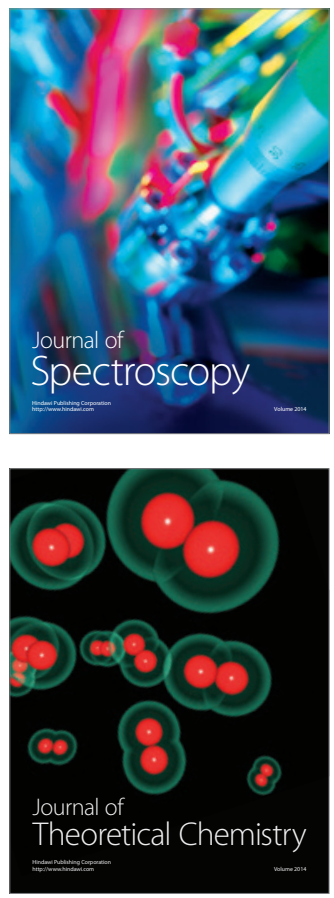
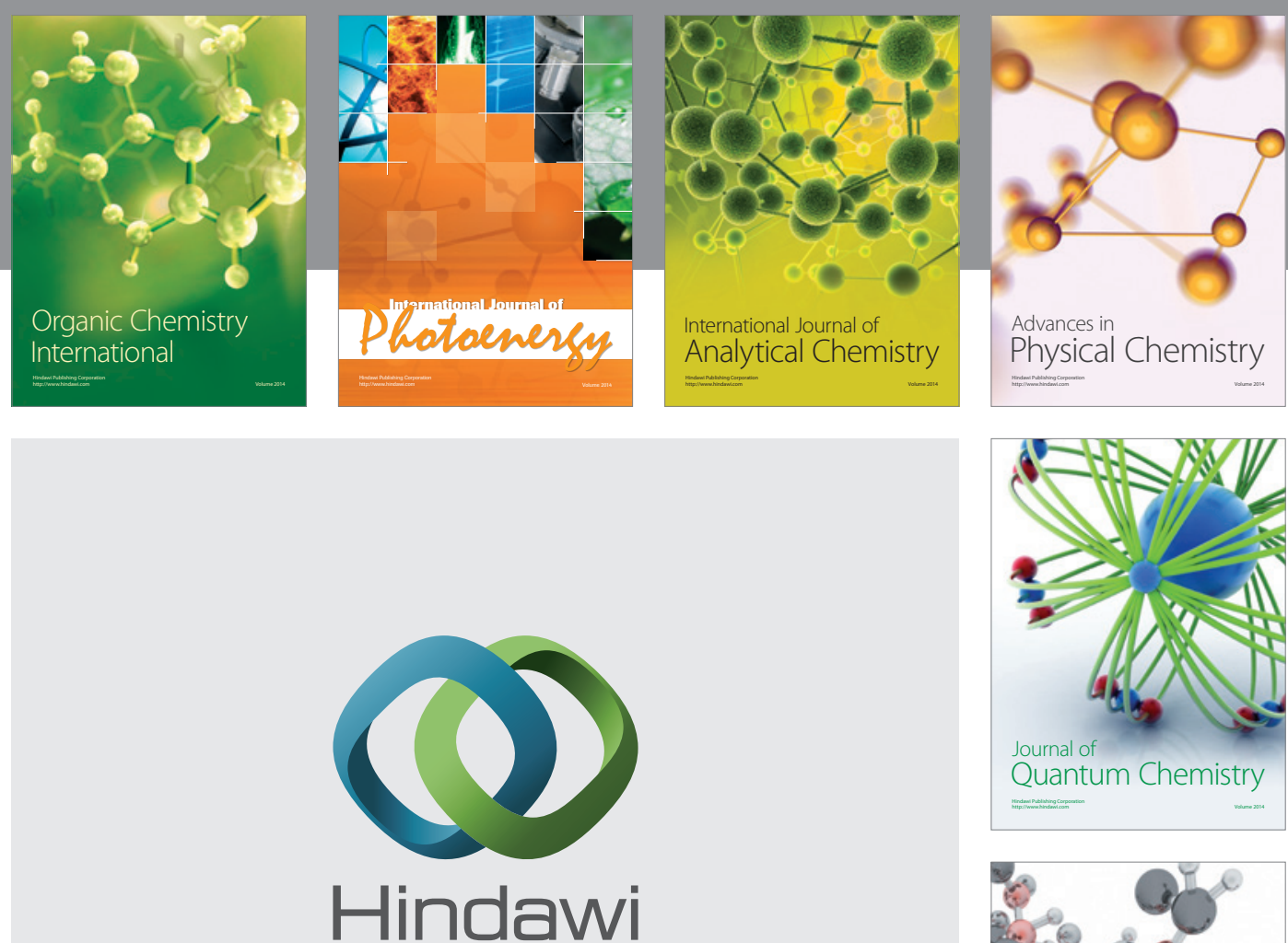

Submit your manuscripts at

http://www.hindawi.com

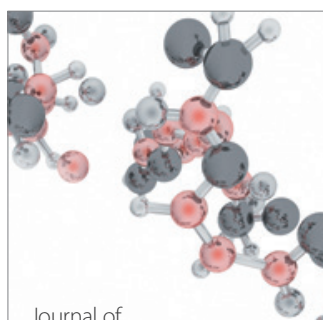

Analytical Methods

in Chemistry

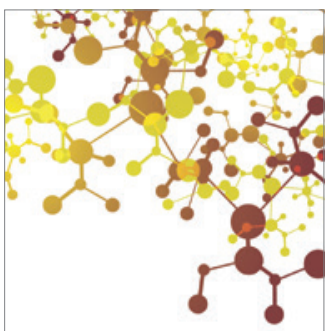

Journal of

Applied Chemistry

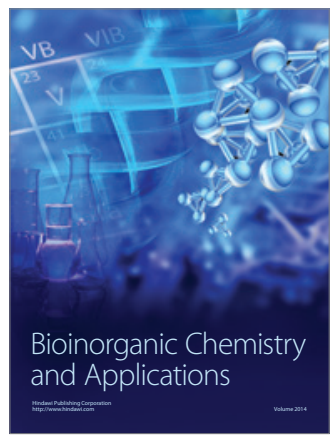

Inorganic Chemistry
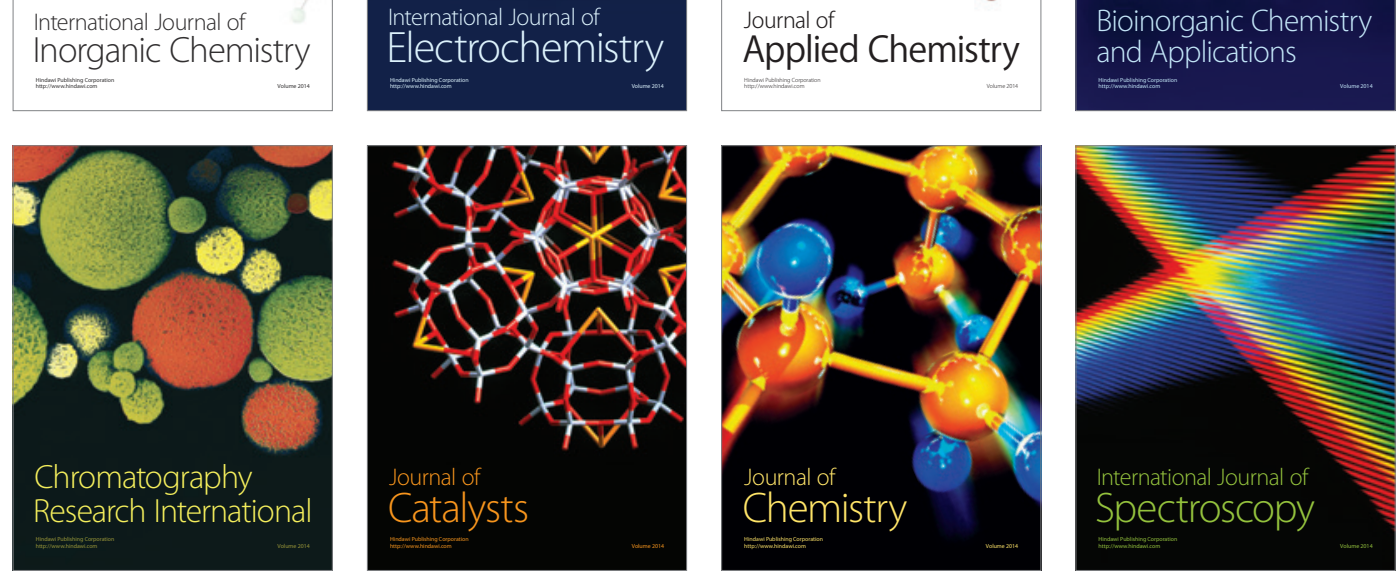